\title{
DOS ESPECIES NUEVAS DE CRASSULACEAE DEL SUR DE JALISCO, MÉXICO
}

\author{
H. David Jimeno-Sevilla ${ }^{1}$, Francisco J. Santana Michel $^{2}$ y Pablo Carrillo- \\ REYES $^{3,4}$
}

${ }^{1}$ Instituto Tecnológico Superior de Zongolica, km 4 Carretera a la Compañía s/n, 95005 Zongolica, Veracruz, México.

${ }^{2}$ Universidad de Guadalajara, Departamento de Ecología y Recursos Naturales, Centro Universitario de la Costa Sur, Independencia Nacional 151, 48940 Autlán, Jalisco, México.

${ }^{3}$ Universidad de Guadalajara, Departamento de Botánica y Zoología, Centro Universitario de Ciencias Biológicas y Agropecuarias, km 15.5 carretera a Nogales, Predio Las Agujas, 45110 Zapopan, Jalisco, México.

4Autor para la correspondencia: pcarreyes@gmail.com

\section{RESUMEN}

Con base en colectas procedentes del sur del estado de Jalisco, se describen Echeveria rulfiana y Villadia ramirezii como especies nuevas para la ciencia. La primera se asigna a la serie Gibbiflorae y se compara con E. marianae, E. novogaliciana y E. nayaritensis, de las que se distingue principalmente por la presencia de tallo y por sus hojas oblongas y acanaladas. V. ramirezii parece estar estrechamente relacionada con $V$. platystyla y se puede separar, entre otros caracteres, por poseer inflorescencias más laxas y flores de menor tamaño. Se discute el estado de conservación de ambas especies, que parecen representar estrechos endemismos en el sur de Jalisco.

Palabras clave: Crassulaceae, Echeveria, endemismo, Gibbiflorae, Villadia.

\begin{abstract}
Based on collections from the southern part of the state of Jalisco, Echeveria rulfiana and Villadia ramirezii are described as new species. The former is assigned to series Gibbiflorae and compared with E. marianae, E. novogaliciana, and E. nayaritensis, from
\end{abstract}


which it differs mainly by its caulescent habit and oblanceolate and canaliculate leaves. The latter is related with $V$. platystyla and can be distinguished by possesing smaller flowers arranged in loose cymes. Both species are narrow endemic elements of southern Jalisco and their conservation statuses are discussed.

Key words: Crassulaceae, Echeveria, endemism, Gibbiflorae, Villadia.

En la porción sur del estado de Jalisco, particularmente en la parte media de la cuenca del Río Ayuquila-Armería, se presenta un sistema de valles y cañadas afectadas por la sombra orográfica (efecto Foehn) producida por la sierra de Manantlán y los Volcanes de Fuego y Nevado de Colima. Esta región representa un enclave que alberga áreas con clima cálido semiárido (BS(h')w) (Martínez et al., 1991). El aislamiento geográfico de la zona podría haber influenciado la especiación de varios linajes de plantas. El fenómeno aparentemente se manifiesta en los numerosos endemismos que esta comarca alberga, entre los que el grupo de las plantas suculentas está bien representado por especies como Bursera macvaughiana Cuevas \& Rzedowski, Euphorbia diazlunana (J.A. Lomelí \& Sahagún) V.W. Steinmann, Echeveria yalmanantlanensis A. Vázquez \& Cházaro y Sedum chazaroi P. Carrillo \& J.A. Lomelí (Lomelí y Sahagún, 1993; Cuevas y Rzedowski, 1999; Carrillo-Reyes y Lomelí-Sención, 2008; Vázquez-García et al., 2013).

Durante diversos viajes de colecta a esta región, así como en el curso de la revisión de ejemplares de herbario para la elaboración del tratamiento de la familia Crassulaceae en el estado de Jalisco, se detectó la existencia de dos especies de Crassulaceae no descritas con anterioridad, las cuales se dan aquí a conocer.

Echeveria rulfiana Jimeno-Sevilla, Santana-Michel \& P. Carrillo sp. nov. Figuras. 1,2 y 3 .

Planta perennis, glabra, caules usque ad $150 \mathrm{~cm}$ longi, 2-2.5 cm diametro. Rosulae laxae, $15-40 \mathrm{~cm}$ diametro, 11-23 foliatae; folia oblanceolata, apice acuminata, mucronata, pseudopetiolata, margine integro, hyalino, $4.5-25 \mathrm{~cm}$ longa, $2-4 \mathrm{~cm}$ lata, cuneata, viridia ad purpurea. Inflorescentia paniculata, 22-69 cm longa, 4-11 cincinis, cum (1)4-12 floribus; bracteae ascendentes, oblanceolatae, acuminatae, $1.5 \mathrm{~cm}$ longae, $4.7 \mathrm{~mm}$ latae, virides; pedicellis turbinatis, $6.7-9 \mathrm{~mm}$ longis, $3 \mathrm{~mm}$ diametro, ferme brateolatis. Sepala lanceolata, apice acuta, inaequalia, patentia ad reflexa. Corolla urceolata, 17-20 $\mathrm{mm}$ longa, $11 \mathrm{~mm}$ diametro basi, pentagonalis, carnea, petalis 
lanceolatis ad oblongo-lanceolatis, ad basin ecavatis et 2 appendicibus dactyloideis 1-1.8 mm longis ornatis; nectariis reniformibus, $3 \mathrm{~mm}$ latis, pallide luteis, stigmata viridia; taxon novum ad seriem Gibbiflorae pertinens; E. novogalicianae J. Reyes, Brachet \& González-Zorzano et E. nayaritensis Kimnach simile.

Planta subarbustiva, glabra, caulescente; tallos ascendentes a colgantes en plantas viejas, de 5 a 40(135) $\mathrm{cm}$ de largo, de 2 a $2.5 \mathrm{~cm}$ de diámetro, de color grisáceo a verde; raíces fibrosas; hojas dispuestas en rosetas laxas, de 15 a $40 \mathrm{~cm}$ de diámetro, con 11 a 23 hojas, láminas oblanceoladas en contorno, acanaladas hacia la parte media, de 4.5 a $25 \mathrm{~cm}$ de largo, de 2 a $4 \mathrm{~cm}$ de ancho, $5 \mathrm{~mm}$ de grosor, con el ápice acuminado, mucronado, ligeramente asimétrico, base más o menos cuneada, pseudopeciolada y espolonada, margen entero, hialino, blanquecino, la cara inferior aquillada, de color verde a verde purpúreo; inflorescencia lateral en panícula, 1 por roseta, de 22 a $69 \mathrm{~cm}$ de alto, con 4 a 11 cincinos, éstos con (1)4 a 12 flores cada uno, pedúnculo de 16 a $48 \mathrm{~cm}$ de largo hasta la primera ramificación, diámetro de la base 5.3 a $10 \mathrm{~mm}$, de color verde; brácteas 11 a 19, alternas, ascendentes, oblanceoladas, caedizas en la fructificación, con la cara superior plana a acanalada y la cara inferior convexa aquillada, de $2.1 \mathrm{a} 12 \mathrm{~cm}$ de largo, 0.6 a $1.8 \mathrm{~cm}$ de ancho, 2.7 a $3 \mathrm{~mm}$ de grosor, de color verde, ápice acuminado con un mucrón pequeño; bractéolas oblanceoladas, de $1.5 \mathrm{~cm}$ de largo y $4.7 \mathrm{~mm}$ de ancho, espolonadas en la base, ápice acuminado, de color verde; pedicelo turbinado, de 6.7 a $9 \mathrm{~mm}$ de largo, $3 \mathrm{~mm}$ de diámetro, de color verde amarillento a rojizo pálido, pruinoso, usualmente bracteolado; bracteolas del pedicelo lanceoladas, de $2.5 \mathrm{~mm}$ de largo, ápice agudo, espolonadas, de color crema; cáliz de 5 sépalos unidos en la base, lanceolados, ápice agudo, desiguales, los mayores de 9 a $10 \mathrm{~mm}$ de largo, 4.4 a $5 \mathrm{~mm}$ de ancho, y los menores de 6 a $7 \mathrm{~mm}$ de largo, 3.7 a $3.8 \mathrm{~mm}$ de ancho, extendidos a reflexos, convexos en ambas caras, de color verde a verde grisáceo, pruinosos; corola urceolada, de 17 a $20 \mathrm{~mm}$ de largo y hasta $11 \mathrm{~mm}$ de diámetro en la base, gibosa, pentagonal, de color rosa anaranjado; segmentos lanceolados a oblongo-lanceolados, unidos en la base formando un tubo de cerca de $2 \mathrm{~mm}$ de largo, segmentos aquillados en su cara externa, de $6 \mathrm{~mm}$ de ancho, ápice agudo, ambos lados de color rosa anaranjado, la parte interna con dos apéndices curvos en la base de los estambres epipétalos, de 1 a $1.8 \mathrm{~mm}$ de largo, de color rosa anaranjado; estambres 10, 5 epipétalos de $1.1 \mathrm{~cm}$ de largo, 5 antisépalos de 1.2 a 1.4 $\mathrm{cm}$ de largo, filamentos de color amarillo pálido, anteras amarillas; nectarios reniformes, de cerca de $3 \mathrm{~mm}$ de ancho de color amarillo pálido; ovario súpero de 5 carpelos libres, de $8 \mathrm{~mm}$ de largo y $4 \mathrm{~mm}$ de ancho, de color amarillo claro, estilo 
de cerca de $8 \mathrm{~mm}$ de longitud, de color rojizo obscuro; estigmas de color verde; folículos maduros ascendentes, de color café rojizo; semillas numerosas, de color café rojizo.

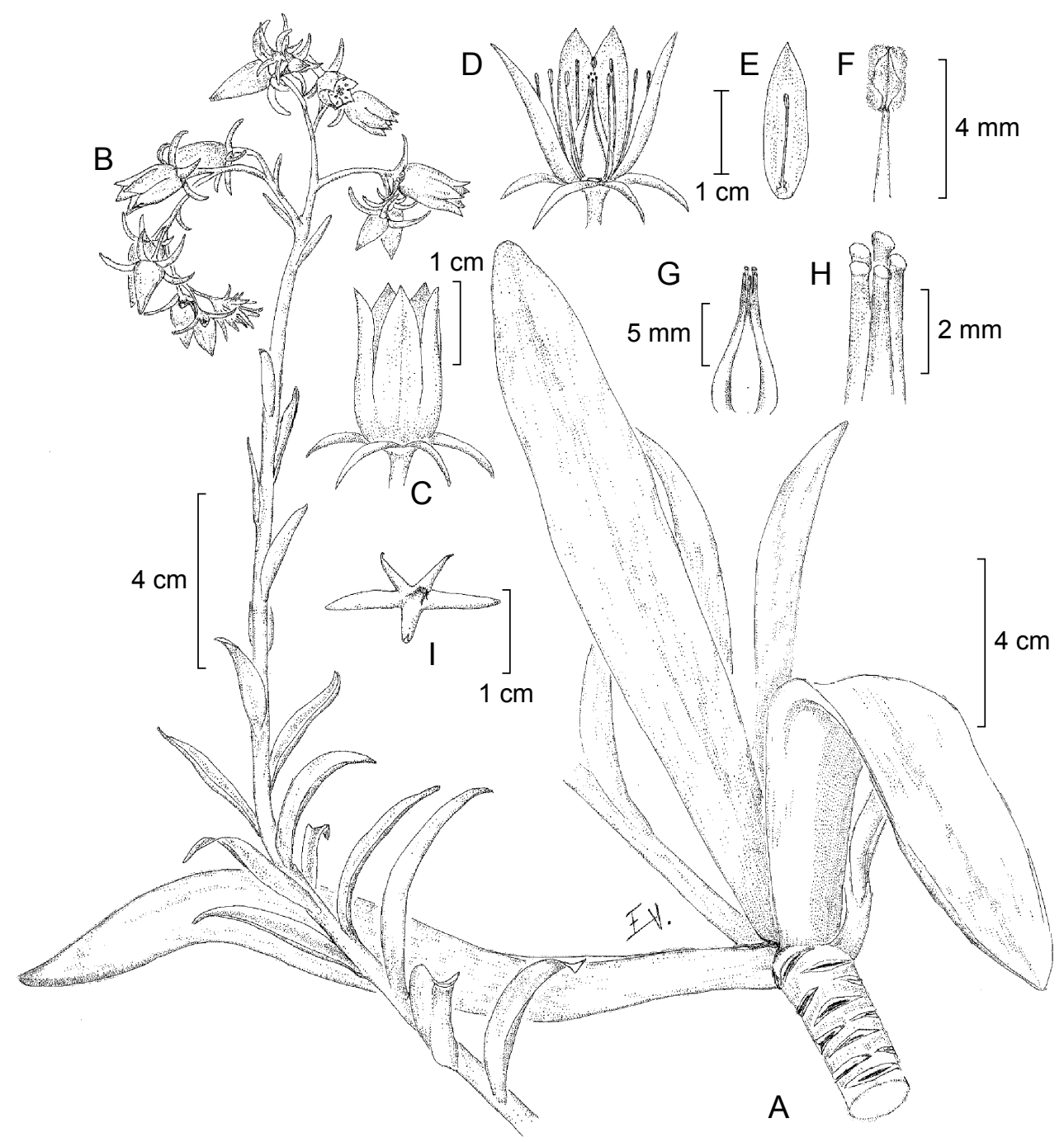

Fig. 1. Echeveria rulfiana. A. hábito; B. inflorescencia; C. flor; D. disección de la flor; E. pétalo; F. detalle de la antera; G. gineceo; H. detalle de los estilos y estigmas; I. cáliz; con base en F. Santana-Michel y E.V. Sánchez 7035 (ZEA). 
Tipo: MÉXICO. Jalisco, mpio. San Gabriel, cerro al oeste de Apango, en cañada sobre rocas ígneas, escasa, 2063 m, 24.XI.2010, D. Jimeno S., F. J. Santana M., D. Vergara R. y M. Castañeda 1336 (Holotipo: IEB; isotipos: IBUG, MEXU, XAL, ZEA).

Paratipos: MÉXICO. Jalisco, Mpio. San Gabriel, $1 \mathrm{~km}$ al O de Apango, $19^{\circ} 48^{\prime} \mathrm{N}, 103^{\circ} 43^{\prime} \mathrm{W}, 1980$ m, 20.XII.2005, P. Carrillo-Reyes y J. A. Lomeli-Sención 5101 (GUADA); San Gabriel, sobre tejados, 1944' 47" N, 10345' 58" W, 1270 m, 19.IV.2011, P. Carrillo-Reyes y J. M. Carrillo 6336 (IBUG, IEB); $1 \mathrm{~km}$ al NE de Apango, 1600 m, 18.XI.2008, M. Cházaro y B. Kemble 9153 (IEB); tejas de las casas de la calle principal del poblado de San Gabriel, 1267 m, 24.XI.2010, D. Jimeno S., F. J. Santana M., D. Vergara R. y M. Castañeda 1338 (XAL); $1 \mathrm{~km}$ al O de Apango camino a Los Llanetes, 1950 m, 7.VI.1992, J. A. Lomeli-Sención y M. Cházaro s.n. (GUADA 23923); Venustiano Carranza (San Gabriel), sobre los tejados, $1350 \mathrm{~m}$, 19.X.1992, L. Vázquez 33 (IEB). Mpio. Tolimán, barranca El Huisichi, ca. $15 \mathrm{~km}$ al S de Tolimán, ladera exp. SO, 800-850 m, 10.X.1997, J. A. Lomelí-Sención et al. 2776 (GUADA); barranca del Huisichi, 15.XI.1992, J. A. Lomeli-Sención s.n. (GUADA 24139). Mpio. Zapotitlán de Vadillo, $5 \mathrm{~km}$ al $\mathrm{N}$ de Zapotitlán por la brecha Copala - Zapotitlán de Vadillo, 1000 m, 12.VIII.1994, F. J. Santana-Michel y E. V. Sánchez R. 6766 (ZEA); $5 \mathrm{~km}$ al $\mathrm{N}$ de Zapotitlán de Vadillo, $1000 \mathrm{~m}, 26 . V I I I .1994, F$. J. Santana-Michel y E. V. Sánchez R. 7035 (ZEA).

Etimología. Se dedica esta especie al ilustre escritor y fotógrafo jalisciense Juan Rulfo (1917-1986), oriundo la ciudad de Sayula, cuya infancia la pasó en el poblado de San Gabriel. Sus trabajos más importantes y por los cuales ha pasado a la historia son "El llano en llamas" (1953) y "Pedro Páramo" (1972).

Distribución y hábitat. Echeveria rulfiana se conoce únicamente de la región sur del estado de Jalisco. Se ha colectado en altitudes de 650 a $2070 \mathrm{~m}$ en bosque de pino y encino, bosque de encino con Acacia, Dodonaea, Eysenhardtia, Ipomoea, Lippia y Tecoma, así como en bosque tropical caducifolio con Acacia, Bursera, Ceiba, Cnidoscolus, Euphorbia, Eysenhardtia, Fouquieria, Hechtia, Jatropha, Lysiloma, Opuntia, Pereskiopsis, Pilosocereus, Pisonia, Plumeria y Stenocereus. Prospera sobre sustratos de origen ígneo y es abundante en los tejados de las casas de algunas localidades, particularmente en San Gabriel, Jalisco.

Fenología. Florece de agosto a noviembre y fructifica de septiembre a diciembre. 

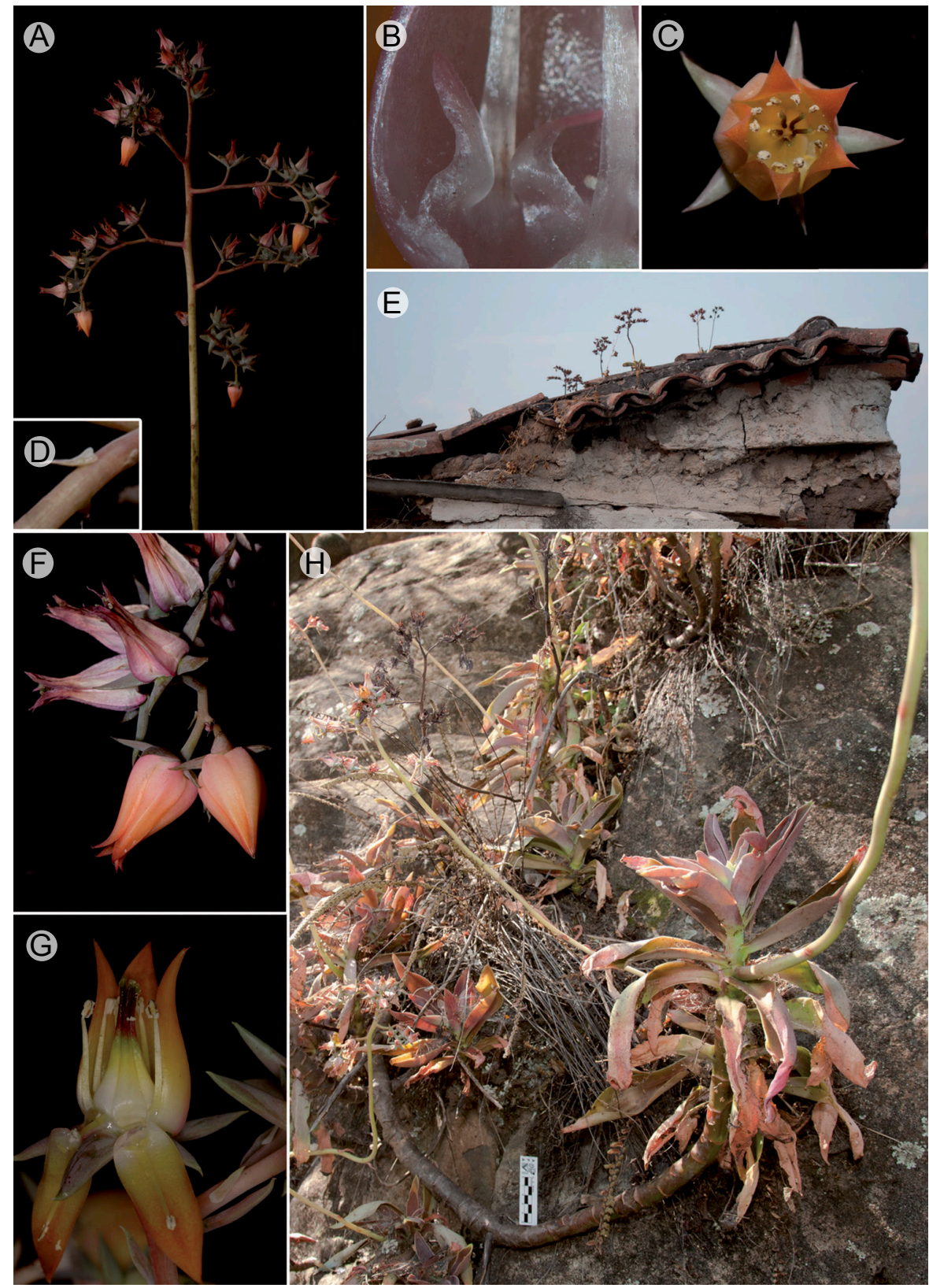

Fig. 2. Echeveria rulfiana. A. inflorescencia; B. apéndices en la base del filamento epipétalo; C. flor vista superior; D. bracteola del pedicelo; E. hábito sobre tejas, poblado de San Gabriel, Jalisco; F. detalle de rama de la inflorescencia; G. detalle de la flor con los pétalos removidos; H. hábito. 
Jimeno-Sevilla et al.: Dos especies nuevas de Crassulaceae, Jalisco, México

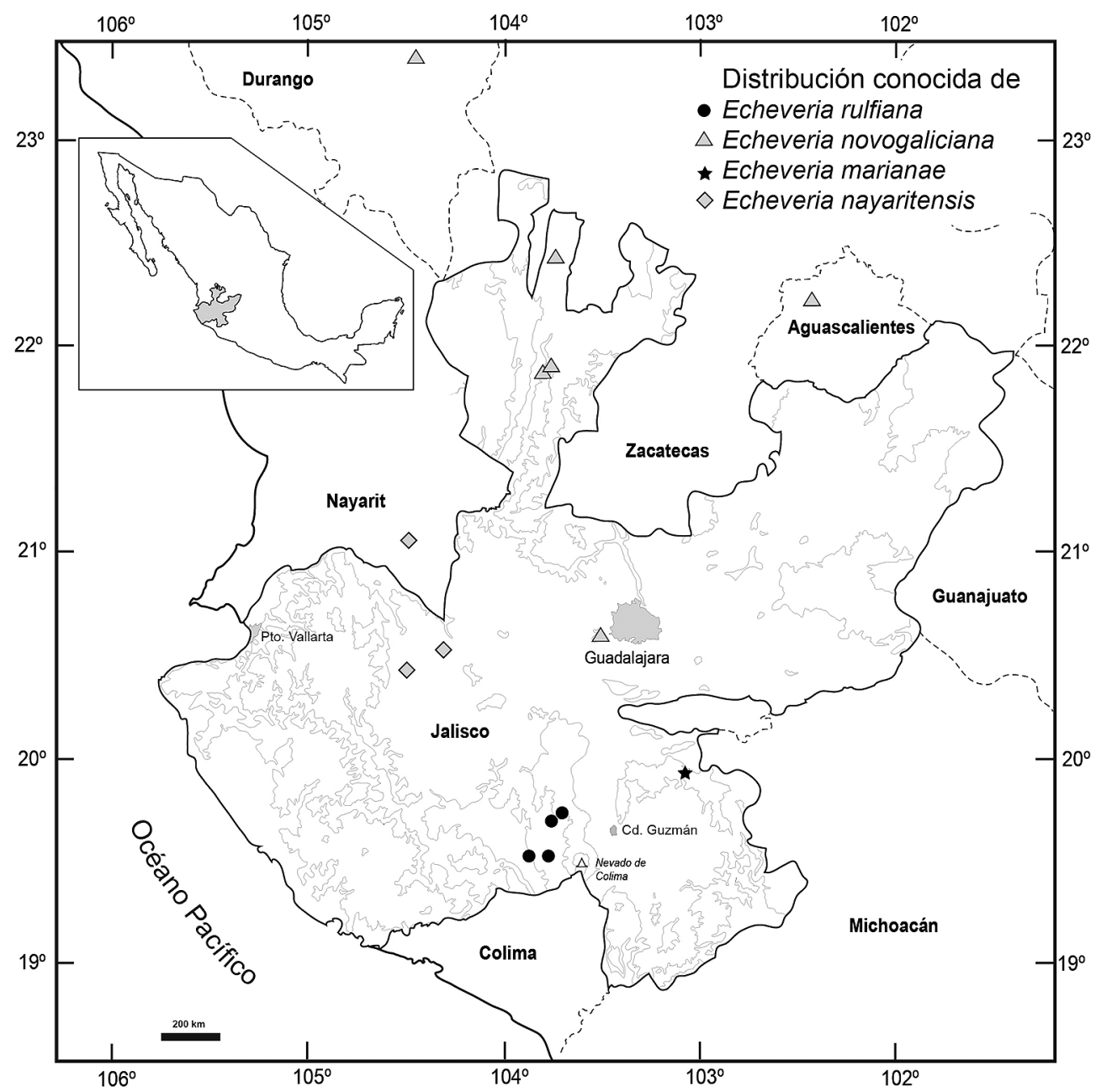

Fig. 3. Mapa de distribución de Echeveria rulfiana y especies afines.

El género Echeveria, cuyo principal centro de distribución se encuentra en México, está conformado por cerca de 140 especies (Walther, 1972; Kimnach, 2003; Pérez-Calix y Franco, 2004), de las cuales 17 se han reportado como silvestres para el estado de Jalisco (Cházaro-Basáñez et al., 2010; Jimeno-Sevilla y Carrillo-Reyes, 2010; Reyes y González-Zorzano, 2010; Vázquez-García et al., 2013; García-Ruiz y Costea, 2014). Con la presente, el número de especies en Jalisco asciende a 18, cinco de las cuales son endémicas al estado. 
Por su hábito robusto y caulescente, ausencia de indumento y por sus inflorescencias en forma de cima paniculada con ramas laterales a manera de cincinos, Echeveria rulfiana se ubica dentro de la serie Gibbiflorae (Baker) A. Berger (Moran, 1974; Kimnach, 2003). Aparentemente las especies más cercanas son E. marianae I. García \& Costea, E. novogaliciana J. Reyes, Brachet \& González-Zorzano y E. nayaritensis Kimnach (Kimnach, 1979; Reyes et al., 2011; García-Ruiz y Costea, 2014), plantas distribuidas en el occidente de México (ver Apéndice), con las que comparte además la presencia de apéndices en la cara interna de los pétalos y el color tinto de la porción apical de los folículos. Echeveria rulfiana no es simpátrica con ninguna de ellas y se separa por la presencia de largos tallos (de hasta $135 \mathrm{~cm}$ ). Comparte además con E. novogaliciana las hojas acanaladas y el color rosado naranja de las flores; sin embargo, en E. rulfiana la corola es urceolada, mientras que en E. novogaliciana es campanulada a ligeramente urceolada. Echeveria nayaritensis es una especie que también posee tallos conspicuos y sépalos reflejos, sin embargo sus hojas son obovadas y planas a cóncavas (vs. oblanceoladas y acanaladas) y es una planta que en general posee menores dimensiones; los tallos más largos de los que se tenga noticia llegan a $22 \mathrm{~cm}$ de largo (vs. $130 \mathrm{~cm}$ ), sus hojas miden 10 a $15 \mathrm{~cm}$ (vs. $4.5-24 \mathrm{~cm}$ ) y sus flores poseen pétalos de $10 \mathrm{a} 15 \mathrm{~mm}$ de largo (vs. $17 \mathrm{a} 20 \mathrm{~mm}$ ). Por último, Echeveria marianae comparte la presencia de flores urceoladas; sin embargo, se distingue de E. rulfiana por la ausencia de tallos, por sus hojas planas de color verde brillante (vs. hojas acanaladas de color verde a verde purpúreo) y por sus flores con pétalos de 18 a $24 \mathrm{~mm}$ de largo (vs. 17 a $20 \mathrm{~mm}$ ) con nectarios rosados (vs. nectarios de color amarillo pálido) (Cuadro 1).

Conservación. Echeveria rulfiana se distribuye en un área reducida y es conocida de unas pocas poblaciones que en sus extremos distan entre sí por aproximadamente $25 \mathrm{~km}$. En una de ellas, la más austral (Santana-Michel y Sánchez 6766; 7035) ha sido aparentemente extinta, debido a la construcción de una carretera asfaltada. Usando la herramienta GeoCAT (Bachman et al., 2011), se calculó que Echeveria rulfiana tiene un área de extensión de la presencia (EOO) de $184.9 \mathrm{~km}^{2} \mathrm{y}$ un área de ocupación (AOO) de $20 \mathrm{~km}^{2}$ considerando celdas de $2 \mathrm{~km}$. Con base en lo anterior, se indica una asignación preliminar a la categoría En Peligro (EN (B2 biii)) en ajuste a los criterios de la IUCN (2001).

Villadia ramirezii P. Carrillo sp. nov. (Figs. 4 y 5).

Planta herbacea perennis, usque $12 \mathrm{~cm}$ alta; radices tuberosas; caules ad basim $4 \mathrm{~mm}$ diametro; folia spiraliter disposita, sessilia, laminis lineari-oblongis, 4-6 
Cuadro 1. Comparación de caracteres distintivos morfológicos, fenología y distribución geográfica entre Echeveria rulfiana y especies aparentemente afines.

\begin{tabular}{|c|c|c|c|c|}
\hline & E. rulfiana & E. marianae & E. novogaliciana & E. nayaritensis \\
\hline Tallo largo $(\mathrm{cm})$ & hasta 135 & $\begin{array}{l}\text { ausente o } \\
\text { inconspicuo }\end{array}$ & ausente o hasta 10 & 13.5 a 22 \\
\hline \multicolumn{5}{|l|}{ Hojas } \\
\hline forma & oblanceoladas & $\begin{array}{l}\text { obovado- } \\
\text { oblanceolada }\end{array}$ & $\begin{array}{l}\text { lanceolado- } \\
\text { ensiforme }\end{array}$ & obovadas \\
\hline pseudopeciolo & presente & ausente & ausente & presente \\
\hline largo $(\mathrm{cm})$ & 4.5 a 25 & 5 a $22(-24)$ & 22 a 27 & $4 \mathrm{a} 10.5$ \\
\hline $\begin{array}{l}\text { ancho }(\mathrm{cm}) \\
\text { borde }\end{array}$ & $\begin{array}{l}2 \text { a } 4 \\
\text { entero, hialino }\end{array}$ & $\begin{array}{l}\text { entero o lobado, } \\
\text { con una línea } \\
\text { delgada y rojiza }\end{array}$ & $\begin{array}{l}3 \text { a } 5 \\
\text { denticulado, } \\
\text { hialino }\end{array}$ & $\begin{array}{l}2 \text { a } 6.5 \\
\text { entero, hialino }\end{array}$ \\
\hline $\begin{array}{l}\text { sección } \\
\text { transversal }\end{array}$ & acanaladas & planas & acanaladas & planas a cóncavas \\
\hline \multicolumn{5}{|l|}{ Corola } \\
\hline forma & urceolada & urceolada & $\begin{array}{l}\text { campanulada } \\
\text { a ligeramente } \\
\text { urceolada }\end{array}$ & campanulada \\
\hline color & $\begin{array}{l}\text { rosa anaranjado } \\
\text { pálido }\end{array}$ & $\begin{array}{l}\text { blanca amarillenta } \\
\text { en la base, naranja } \\
\text { el resto }\end{array}$ & $\begin{array}{l}\text { rosado amarillento } \\
\text { a naranja pálido }\end{array}$ & $\begin{array}{l}\text { blanca amarillenta } \\
\text { en la base, naranja } \\
\text { en el ápice }\end{array}$ \\
\hline Pétalos largo (mm) & 17 a 20 & 15 a 20 & 18 a 24 & 10 a 15 \\
\hline Nectarios, color & amarillo pálido & $\begin{array}{l}\text { rosado con } \\
\text { márgenes rojizos }\end{array}$ & amarillentos & amarillos \\
\hline Floración & $\begin{array}{l}\text { agosto a } \\
\text { noviembre }\end{array}$ & agosto a octubre & $\begin{array}{l}\text { agosto a } \\
\text { noviembre }\end{array}$ & $\begin{array}{l}\text { noviembre a } \\
\text { febrero }\end{array}$ \\
\hline $\begin{array}{l}\text { Distribución } \\
\text { geográfica } \\
\text { conocida }\end{array}$ & sur de Jalisco & sureste de Jalisco & $\begin{array}{l}\text { Aguascalientes y } \\
\text { centro-norte de } \\
\text { Jalisco }\end{array}$ & $\begin{array}{l}\text { sur de Nayarit y } \\
\text { centro-occidente } \\
\text { de Jalisco }\end{array}$ \\
\hline
\end{tabular}

$\mathrm{mm}$ longis, 1-1.5 mm latis; flores in cymis cum 2-6 cincinnis usque 10(12)-floris ordinati, sessiles, albi; calyx 5 sepalis subaequalibus, usque $1.9 \mathrm{~mm}$ longis, ca. $1 \mathrm{~mm}$ latis; petalis 5, triangularibus, usque $3.4 \mathrm{~mm}$ longis, $1.6 \mathrm{~mm}$ latis; nectariis cuadrangularibus ca. $0.6 \mathrm{~mm}$ longis et latis; follicula $2.2 \mathrm{~mm}$ lata.

Planta herbácea perenne, suculenta, glabra, rupícola, de hasta $12 \mathrm{~cm}$ de alto (incluyendo la inflorescencia); raíces tuberosas. Tallos estériles de hasta $1 \mathrm{~cm}$ de 
largo, $4 \mathrm{~mm}$ de diámetro en la base; rosetas de ca. $1.5 \mathrm{~cm}$ de diámetro; hojas ca. 15 , linear-oblongas, 4 a $6 \mathrm{~mm}$ de largo y 1 a $1.5 \mathrm{~mm}$ de ancho, ápice agudo; tallos floríferos de 3.5 a $6 \mathrm{~cm}$ de largo y 0.1 a $0.15 \mathrm{~cm}$ de diámetro en la base; inflorescencia de tipo cimoso de (1)3 a $6 \mathrm{~cm}$ de largo y (1.5-) 4 a $5 \mathrm{~mm}$ de ancho, con un eje central y 2 a 6 ramas secundarias en forma de cincinos, cada una con 3 a 10(12) flores; brácteas y bractéolas similares a las hojas; brácteas de (4-)8-11.5 mm de largo y 0.9 a $1.2 \mathrm{~mm}$ de ancho; bractéolas de $3.7 \mathrm{~mm}$ de largo y ca. $1.1 \mathrm{~mm}$ de ancho; flores sésiles, de 2.5-3 $\mathrm{mm}$ de largo y ca. $2 \mathrm{~mm}$ de diámetro, cáliz con 5 lóbulos subiguales, lanceolados, verdes, de hasta $1.9 \mathrm{~mm}$ de largo y ca. $1 \mathrm{~mm}$ de ancho en la base, papilosos hacia el ápice; corola glabra, pétalos 5, blancos, triangulares, con el ápice agudo, de 2.6 a $3.4 \mathrm{~mm}$ de largo y $1.6 \mathrm{~mm}$ de ancho en la base, ascendentes, incurvados, formando un tubo de 0.5-0.8 mm; estambres 10, glabros, 5 de ellos antisépalos y adnados a los pétalos, de ca. $1.2 \mathrm{~mm}$ de largo, con los filamentos triangulares de $0.6 \mathrm{~mm}$ de largo y ca. $0.4 \mathrm{~mm}$ de ancho en la base, los otros 5 antipétalos, de ca. $2.2 \mathrm{~mm}$ de largo, filamentos blancos, anteras de ca. 0.6 $\mathrm{mm}$ de largo y 0.4 a $0.5 \mathrm{~mm}$ de ancho, suborbiculares; nectarios cuadrangulares, de ca. $0.6 \mathrm{~mm}$ de alto y ancho, de color crema; gineceo glabro, de $1.6 \mathrm{~mm}$ de alto y ca. $1.5 \mathrm{~mm}$ de ancho, carpelos connados en la base por ca. $0.3 \mathrm{~mm}$, cada carpelo de ca. $0.9 \mathrm{~mm}$ de ancho; folículos de hasta $2.2 \mathrm{~mm}$ de alto y $1 \mathrm{~mm}$ de ancho en fructificación, con numerosas semillas.

Tipo: MÉXICO. Jalisco, Mpio. San Gabriel, km 20 camino Tapalpa - San Gabriel. (aprox. $4.5 \mathrm{~km}$ en línea recta al NO de Jiquilpan), 19²48'58" N, 1034ㅇ' W, 1880 m, bosque de encino con Heliocarpus, Lysiloma, 25.VIII.2004, P. CarrilloReyes y A. Kennedy 4417 (Holotipo: IBUG, isotipo: IEB).

Paratipos: MÉXICO. Jalisco, Mpio. Autlán, 1-2 km al W de Ahuacapán, 1400 m, 3.X.1991. F.J. Santana-Michel et al. 5442 (ZEA); Mpio. San Gabriel, 6-7 km después de San Gabriel rumbo a Tapalpa, 1900 m, 28.IX.1991. M. Huerta et al. 165 (IEB, XAL).

Etimología. El nombre de la especie honra al recientemente desaparecido Ing. Raymundo Ramírez Delgadillo (1963-2011), del Instituto de Botánica de la Universidad de Guadalajara, quien fuera un prolífico colector botánico, un dedicado profesor dentro y fuera de las aulas y sobre todo, un querido amigo.

Nombre común. "Flor de piedra" (Santana-Michel et al. 5442). 

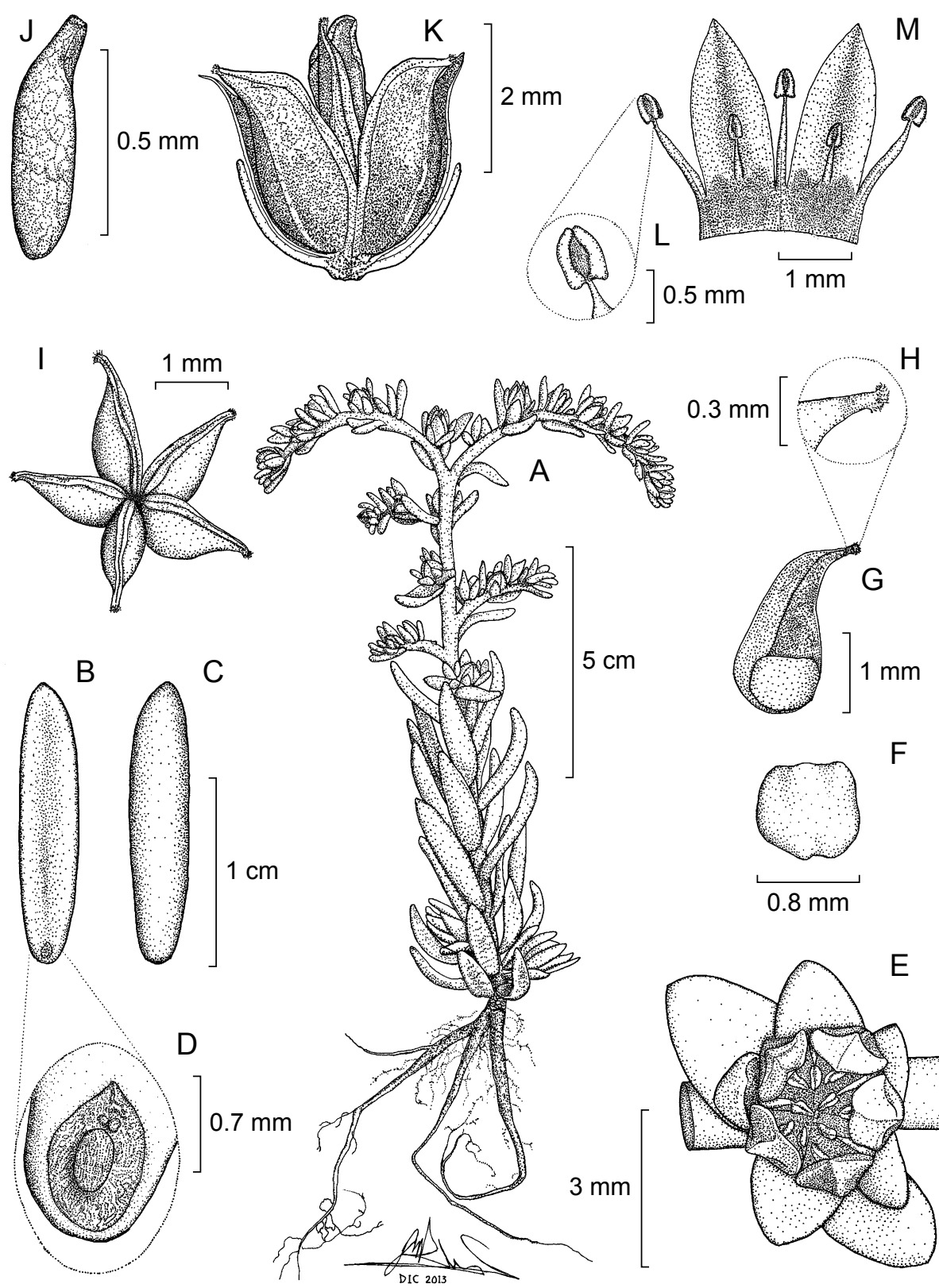

Fig. 4. Villadia ramirezii. A. hábito; B. hoja en vista adaxial; C. hoja en vista abaxial; D. detalle de la inserción de la hoja; E. flor en vista radial; F. escama nectarífera; G. carpelo; H. estigma; I. gineceo en vista radial; J. semilla; K. gineceo con dos carpelos removidos para mostrar la fusión de los mismos; L. antera; M. detalle de la parte interna de la corola. 
Distribución y habitat. Se conoce de dos localidades en el sur del estado de Jalisco, una de ellas en los límites de los municipios de San Gabriel y Tapalpa, en la estribación sur de la Sierra de Tapalpa y la otra en el municipio de Autlán, en la vertiente norte de la Sierra de Manantlán. Se ha colectado entre 1400 y $1900 \mathrm{~m}$ de altitud sobre sustrato de origen ígneo en bosque de encino con algunos elementos de bosque tropical caducifolio.

Fenología. Florece en septiembre y octubre.

Villadia Rose es un género de aproximadamente 22 especies distribuidas de manera disyunta desde el sur de los Estados Unidos de América hasta Guatemala y en Perú (Thiede, 2003). Comprende plantas con corolas de pétalos unidos al menos en la base y típicamente de inflorescencias en forma de espiga o tirso. Su delimitación ha estado sujeta a algunas controversias (Fröderström, 1935; Baehni, 1937; Walther, 1938; Clausen, 1940), originadas principalmente por la incierta relación con un grupo de especies de corolas también tubulares, pero de inflorescencias cimosas que fueron reconocidas originalmente en Altamiranoa (Britton y Rose, 1903) y que más recientemente fueron transferidas a Sedum (Moran, 1996). El concepto original de Villadia (Britton y Rose, 1903) es ampliamente seguido en la actualidad y existe vasto consenso respecto a su delimitación (Thiede, 2003; Thiede y Eggli, 2007; Pérez-Calix, 2008). Sin embargo, hipótesis filogenéticas generadas mediante el análisis de marcadores moleculares (Carrillo-Reyes et al., 2009) sugieren que $V i$ lladia es un conjunto parafilético y que el tipo de inflorescencia tirsoide ha aparecido en múltiples ocasiones dentro del "Grupo Villadia" y no es un carácter diagnóstico para el reconocimiento del género. La circunscripción de Villadia y Altamiranoa debe ser reevaluada con base en evidencia filogenética.

Villadia ramirezii se agrupa en el citado análisis como especie hermana de $V$. platystyla (Fröd.) R.T. Clausen (otra planta con inflorescencia en forma de cimas), dentro de un clado bien soportado que comprende a varios elementos del norte y centro de México, todos con inflorescencias tirsoides. Dado que este clado incluye a $V$. misera (Lindl.) R.T. Clausen, especie tipo del género, se decidió ubicar al taxon nuevo en el género Villadia en lugar de hacerlo en Sedum o en Altamiranoa. Villadia platystyla y $V$. ramirezii son los únicos componentes de Villadia sensu stricto con inflorescencia en forma de cimas.

Villadia ramirezii está estrechamente relacionada con $V$. platystyla, también endémica al estado de Jalisco; sin embargo, la especie nueva es de una planta menos robusta con flores de menores dimensiones. Villadia ramirezii se distingue por 


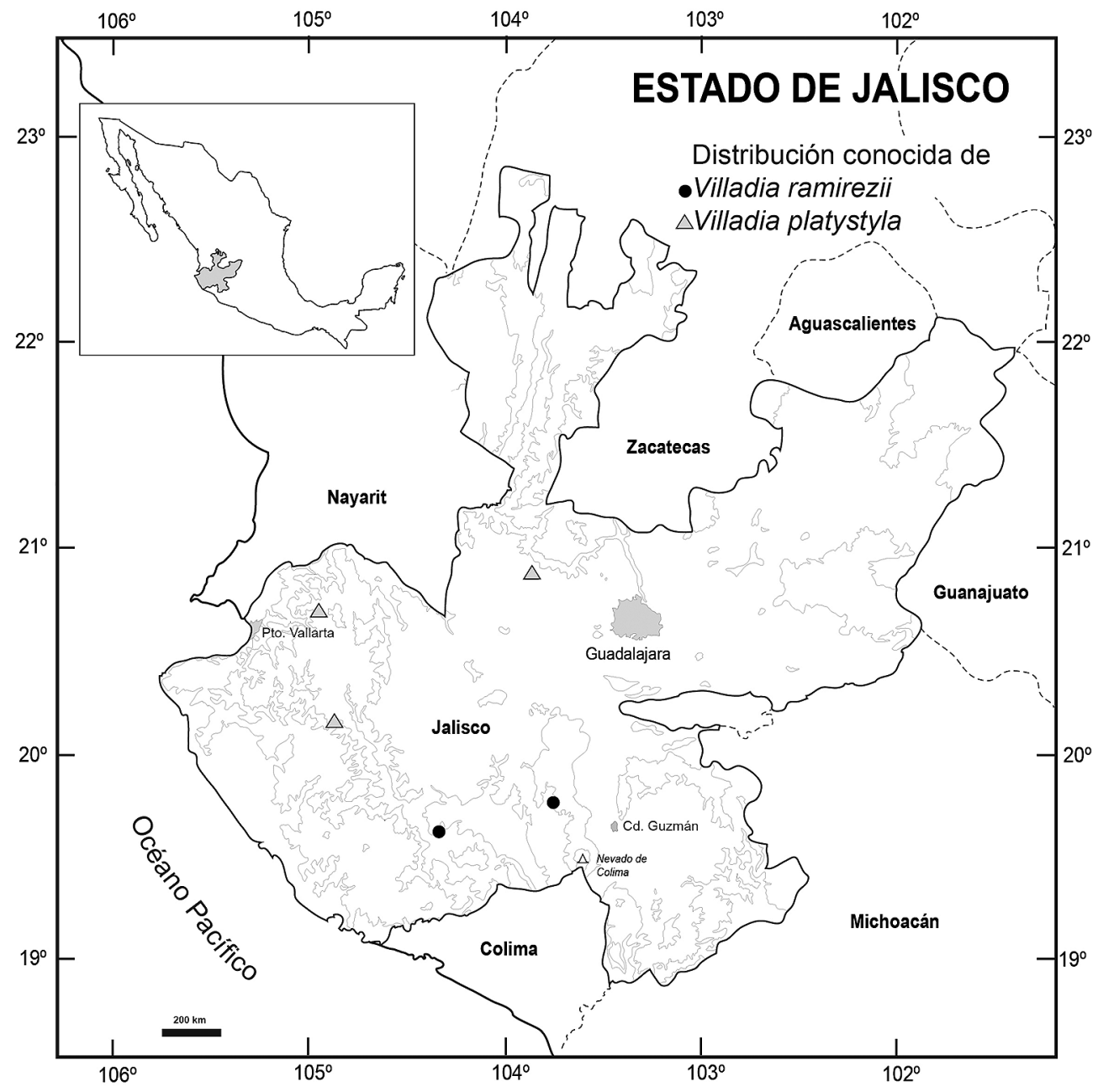

Fig. 5. Mapa de distribución de Villadia ramirezii y V. platystyla.

poseer inflorescencias laxas con brácteas de hasta $1.2 \mathrm{~mm}$ de ancho (vs. $3-5 \mathrm{~mm}$ de ancho), flores separadas entre sí por (2-)3-3.5 mm (vs. distancia entre flores menor a $2 \mathrm{~mm}$ ) y flores pequeñas de hasta $3 \mathrm{~mm}$ de largo (vs. flores de hasta $5 \mathrm{~mm}$ de largo). Adicionalmente ambas especies se encuentran separadas ecológica y fenológicamente: $V$. ramirezii solo se conoce de altitudes entre los 1400 y $1900 \mathrm{~m}$ del ecotono del encinar con el bosque tropical caducifolio y se ha encontrado con flores en septiembre y octubre, mientras que Villadia platystyla solo se ha registrado de bosques de 
pino y encino, por arriba de los $2200 \mathrm{~m}$ de altitud y florece de noviembre a enero (ver Apéndice). En el Cuadro 2 se muestran diferencias entre $V$. ramirezii y $V$. platystyla.

Conservación. Se conoce únicamente de dos localidades en el Sur de Jalisco en los municipios de Autlán y San Gabriel. La población del municipio de Autlán se encuentra en el límite de la reserva de la biosfera de la Sierra de Manantlán en una zona en la que no se han observado amenazas aparentes a su conservación, por otra parte, la del municipio de San Gabriel se encuentra aledaña al camino que conduce de San Gabriel a Tapalpa, mismo que fue recientemente ampliado y pavimentado. Un intento de relocalizar estas plantas en abril de 2011, poco después de la mencionada modificación a la vía, resultó infructuoso. Aparentemente no existen colectas posteriores en el área y el estatus de tal población no ha sido evaluado desde entonces. Usando la herramienta GeoCAT (Bachman et al., 2011), se calculó que Villadia ramirezii tiene un área de extensión de la presencia (EOO) de $4.29 \mathrm{~km}^{2}$ y un área de ocupación (AOO) de $8 \mathrm{~km}^{2}$ basada en celdas de $2 \mathrm{~km}$. Con fundamento en lo anterior, se indica una signación preliminar de En Peligro Crítico (CR (B2 biii)) con base en los criterios de la IUCN (2001).

Cuadro 2. Comparación entre Villadia ramirezii y V. platystyla.

\begin{tabular}{|c|c|c|}
\hline & V. ramirezii & V. platystyla \\
\hline \multicolumn{3}{|l|}{ Hojas } \\
\hline forma & linear-oblongas & obovadas \\
\hline largo (mm) & 4 a 6 & 8 a 13 \\
\hline ancho $(\mathrm{mm})$ & 1 a 1.5 & 3 \\
\hline \multicolumn{3}{|l|}{ Brácteas } \\
\hline largo (mm) & (4-)8 a 11.5 & (3-)7 a 12 \\
\hline ancho (mm) & 0.9 a 1.2 & 3 a 5 \\
\hline Distancia entre las flores (mm) & $(2-) 3$ a 3.5 & 0.5 a 2 \\
\hline \multicolumn{3}{|l|}{ Sépalos } \\
\hline largo (mm) & 1.9 & (3-) $5-5.5$ \\
\hline ancho (mm) & 1 & $2-2.1$ \\
\hline \multicolumn{3}{|l|}{ Pétalos } \\
\hline largo (mm) & 3.4 & 5 \\
\hline ancho $(\mathrm{mm})$ & 1.6 & 2 \\
\hline Vegetación & $\begin{array}{l}\text { bosque de encino y ecotono } \\
\text { con el bosque tropical } \\
\text { caducifolio }\end{array}$ & bosque de pino y encino \\
\hline Altitud (m) & $1400-1900$ & $2200-2900$ \\
\hline
\end{tabular}




\section{AGRADECIMIENTOS}

Agradecemos a Juan Manuel Carrillo García, Arturo Castro Castro, Miguel Castañeda, Roberto Castro, Miguel Cházaro Basáñez, Aarón H. Kennedy, Rosa Murguía y Daniela Vergara por su ayuda y compañía para realizar el trabajo de campo; a los curadores de los herbarios BM, CAS, CORU, IBUG, IEB, MEXU, MO, TEX, WIS, XAL, XALU y ZEA, por permitirnos revisar sus ejemplares; a Enrique Valente Sánchez Rodríguez por la ilustración de Echeveria rulfiana y a José Manuel Ramírez Amezcua por la de Villadia ramirezii. Los comentarios de tres revisores anónimos ayudaron a mejorar considerablemente el trabajo.

\section{LITERATURA CITADA}

Baehni, C. 1937. Villadia et Altamiranoa: Étude sur la fusion de deux genres de Crassulacées. Candollea 7: 283-286.

Bachman, S. J. Moat, A. Hill, J. de la Torre y B. Scott. 2011. Supporting Red List threat assessments with GeoCAT: geospatial conservation assessment tool. ZooKeys 150: $117-126$.

Britton, N. L. y J. N. Rose. 1903. New or noteworthy North American Crassulaceae. Bull. New York Bot. Gard. 3: 1-45.

Carrillo-Reyes, P. y J. A. Lomelí-Sención. 2008. Sedum chazaroi (Crassulaceae), an endemic new species from southern Jalisco, Mexico. Bol. Soc. Bot. Mex. 83: 77-80.

Carrillo-Reyes, P., V. Sosa y M. E. Mort. 2009. Molecular phylogeny of the Acre clade (Crassulaceae): dealing with the lack of definitions for Echeveria and Sedum. Mol. Phyl. Evol. 53: 267-276.

Cházaro-Basáñez, M., J. Acevedo-Rosas, R., H. D. Jimeno-Sevilla y O. Zuno-Delgadillo. 2010. Las crasuláceas de Jalisco (México): Listado actualizado. Bol. Soc. Latin. Carib. Cact. Suc. 7: 26-29.

Clausen, R. T. 1940. Studies in the Crassulaceae: Villadia, Altamiranoa and Thompsonella. Bull. Torr. Bot. Club 67: 195-198.

Cuevas, R. y J. Rzedowski. 1999. Una especie nueva de Bursera (Burseraceae) del occidente de México. Acta Bot. Mex. 46: 77-81.

Fröderström, H. 1935(1936). The genus Sedum L. A systematic essay. Part IV. Acta Horti Gothob. 10 (App.): 1-262.

García-Ruiz, I. y M. Costea. 2014. Echeveria marianae (Crassulaceae), a new species from Jalisco, México. Phytotaxa 170: 35-40.

IUCN. 2001. Red List Categories and Criteria: Version 3.1. International Union for Conservation of Nature. http://www.iucnredlist.org/technical-documents/categoriesand-criteria/2001-categories-criteria. Consultado en julio 2014. 
Jimeno-Sevilla, H. D. y P. Carrillo-Reyes. 2010. Echeveria perezcalixii (Crassulaceae), una especie nueva del occidente de México. Brittonia 62: 303-308.

Kimnach, M. 1979. A new Echeveria from Nayarit, Mexico. Cact. Suc. J. (Los Angeles) 51: 207-209.

Kimnach, M. 2003. Echeveria. In: Eggli, U. (ed.). Illustrated handbook of succulent plants: Crassulaceae. Springer-Verlag. Berlín, Alemania. pp. 103-128.

Lomelí, J. A. y E. Sahagún. 1993. Pedilanthus diazlunanus (Euphorbiaceae), especie nueva del sur de Jalisco, México. Acta Bot. Mex. 25: 15-20.

Martínez, L. M., J. J. Sandoval y R. D. Guevara. 1991. El clima en la Reserva de la Biosfera Sierra de Manantlán (Jalisco-Colima, México) y en su área de influencia. Agrociencia Ser. Agua-Suelo-Clima 2: 107-119.

Meyrán, J. y L. López. 2003. Las crasuláceas de México. Sociedad Mexicana de Cactología, A.C. México, D.F., México. 234 pp.

Moran, R. 1974. Echeveria. In: Jacobsen, H. (ed.). Lexicon of succulent plants. Bland-ford Press. Londres, UK. pp. 184-186.

Moran, R. 1996. Altamiranoa into Sedum. Haseltonia 4: 46.

Pérez-Calix, E. e I. S. Franco. 2004. Crasuláceas. In: García-Mendoza, A. J., M. J. Ordóñez y M. Briones-Salas (eds.). Biodiversidad de Oaxaca. Instituto de Biología, Universidad Nacional Autónoma de México - Fondo Oaxaqueño para la Conservación de la Naturaleza - World Wildlife Fund. México, D.F., México. pp. 209-217.

Pérez-Calix, E. 2008. Crassulaceae. Flora del Bajío y de Regiones Adyacentes 156: 1-141.

Pilbeam, J. 2008. The genus Echeveria. The British Cactus \& Succulent Society. Essex, UK. 333 pp.

Reyes, J. y O. González. 2010. Echeveria roseiflora (Crassulaceae) una nueva especie para el estado de Jalisco, México. Cact. Suc. Mex. 55: 19-26.

Reyes, J., C. Brachet y O. González. 2011. Echevera novogaliciana, una nueva especie de la familia Crassulaceae para los estados de Aguascalientes y Jalisco, México. Cact. Suc. Mex. 56:82-95.

Thiede, J. 2003. Villadia. In: Eggli, U. (ed.). Illustrated handbook of succulent plants: Crassulaceae. Springer-Verlag. Berlín, Alemania. pp. 367-374.

Thiede, J. y U. Eggli. 2007. Crassulaceae. In: Kubitzki, K. (ed.). The families and genera of vascular plants. Vol. 9. Springer. Hamburg, Alemania. pp. 83-118.

Vázquez-García, J. A., D. Jimeno-Sevilla, R. Cuevas-Guzmán, M. Cházaro-Basáñez y M. A. Muñiz-Castro. 2013. Echeveria yalmanantlanensis (Crassulaceae): A new species from Cerro Grande, Sierra de Manantlán, western Mexico. Brittonia 65: 273-279.

Walther, E. 1938. Notes on Crassulaceae. Cact. Succ. J. (Los Angeles) 10: 22-24.

Walther, E. 1972. Echeveria. California Academy of Sciences. San Francisco, USA. 426 pp. 


\section{APÉNDICE}

Ejemplares selectos de Echeveria nayaritensis, E. novogaliciana y Villadia platystyla usados para la comparación morfológica con $E$. rulfiana y $V$. ramirezii respectivamente.

Echeveria nayaritensis Kimnach

MÉXICO. Jalisco: Mpio. Ameca, 16 miles northwest of Ameca along the road to Mascota, 4300 ft. 1.XI.1970, D. E. Breedlove 18662 (CAS); km. 29 carretera Ameca - Talpa de Allende, 1550 m, 20.XI.1997, M. Cházaro et al. $7773 B$ (IEB); km 27.5 carretera de Ameca a Talpa de Allende, 11.XII.1994, J. A. Machuca 7268 (MEXU). Mpio. Atenguillo, Balneario El Limón, 20²5'47" N, 104³1'45" W, 1490 m, 13.XI.2012, P. Carrillo-Reyes y A. Castro-Castro 6898 (IBUG); ibid. $1480 \mathrm{~m}$, 2.X.1999, J. A. Lomeli-Sención \& R. Rivera 3095 (GUADA, MEXU). Nayarit: Mpio. Ahuacatlán, brecha entre Barranca del Oro y El Rosario, 9.IX.1990, J. A. Machuca y M. Cházaro $6557 a$ (IEB, MEXU); km. 16.7 carretera Ahuacatlán a Barranca del Oro, 1700 m, 26.XI.1995, J. A. Machuca y M. Cházaro 7642 (IBUG, IEB, MEXU).

Echeveria novogaliciana J. Reyes, Brachet \& González-Zorzano

MÉXICO. Durango: Mpio. Mezquital, km. 3.4 del camino San Miguel Temohaya - El Troncón (aprox. $2 \mathrm{~km}$ al NNE de San Miguel Temohaya), 2319'03" N, 104²9'37" W, 1590 m, 16.X.2005, P. Carrillo-Reyes y E. Ruiz 4839 (IBUG, IEB); a $3.5 \mathrm{~km}$ de Temoaya, 23ำ' N, 104²9' W, $1500 \mathrm{~m}$, M. González 1281 (IBUG). Jalisco: Mpio. Bolaños, $23 \mathrm{~km}$ al NO de Bolaños, estación de microondas El Astillero, 2152'16" N, 10350'26" W, 1600 m, 24.X.1996, J. Calónico-Soto y J. Flores 3268 (MEXU); predio Las Berenjenas, $10 \mathrm{~km}$ al NO de Bolaños, 21 ${ }^{\circ} 52.76^{\prime}$ N, 10351.77' W, 30.XI.2002, E. Domínguez 38 (MEXU). Mpio. Mezquitic, Bajío Huatza Mayeve, 2000 m, 15.XI.1985, C. Chávez s.n. (IBUG 62397). Mpio. Zapopan, cima del cerro del Colli, 3.X.1990, 1800-1900 m, M. Cházaro y R. Ramírez 6427 (IEB); cerro del Colli, 1760 m, 3.X.1991, M. Cházaro et al., 6765 (MO); ibid. 2.XI.1990, M. Cházaro y A. Fayad $6466 a$ (IEB); cerro del Colli, $2 \mathrm{~km}$ al SE de Cd. Granja, $1850 \mathrm{~m}, 2 . X .1993$, G. González et al., 95 (IBUG); cerro del Colli, 1800 m, 21.I.1986, R. Ramírez-Delgadillo y R. Soltero 844 (IBUG). 
Villadia platystyla Fröd.

MÉXICO. Jalisco: Mpio. San Sebastián del Oeste, Real Alto, La Bufa, 2500 m, 30.I.1927, Y. Mexia 1594 (BM, CAS, MO). Mpio. Talpa de Allende, $19 \mathrm{~km}$ al NE de El Cuale, cima del cerro La Tetilla, 20²2'37" N, 10458'38.6" W, 2260 m, 29.XI.2009, A. Castro-Castro et al. 2025 (IBUG); km 30 camino Talpa de Allende a El Cuale, extremo O del cerro San Pedro, $5 \mathrm{~km}$ al SO de la Tetilla de El Cuale, 20²1'23" N, 10500'41" W, 2481 m, 18.III.2013, L. Hernández et al. s.n. (IBUG). Mpio. Tequila, cima del Volcán de Tequila, 2850 m, 7.XII.1986, M. Cházaro y E. de la Mora 4202 (IBUG, WIS); ibid. M. Cházaro y J. Taylor 6469 (IEB); ibid. 13.XII.2002, M. Cházaro y J. L. Álvarez 8238 (IEB); summit of La Tetilla, top of Volcano Tequila, 2950, 14.I.1990, M. Cházaro et al. 6172 (TEX); cima del Volcán de Tequila, 2920 m, 28.III.1987, A. Rodríguez y R. Guzmán 680-a (IBUG); Cima del Volcán de Tequila, 2900 m, 11.I.2003, G. López-Damián y M. Cházaro 31 (IBUG, IEB); parte alta del volcán de Tequila, entre las antenas de microondas y La Tetilla, $20^{\circ} 47^{\prime} 15^{\prime \prime} \mathrm{N}, 103^{\circ} 50^{\prime} 40^{\prime \prime} \mathrm{W}, 2875$ m, 7.I.2011, P. Carrillo-Reyes y A. Castro-Castro 6222 (IBUG, IEB). 